\title{
A LEAK-TIGHT AND ZERO-DEAD-VOLUME MICRO GAS PUMP INTEGRATED WITH ELECTROKINETIC LIQUID PISTONS \\ J.Jiao $^{* 1}$, R. Almeida ${ }^{l}$, and J.W. Kwon ${ }^{l}$ \\ ${ }^{1}$ Department of Electrical and Computer Engineering, University of Missouri, Columbia, MO, U.S.A.
}

\begin{abstract}
This paper presents a novel micromachined gas pump utilizing electrokinetically driven liquid pistons in a microfluidic channel. As a result of our effort in improving the leakage problem of micro gas pumps, we have successfully utilized a low contact angle liquid droplet as a piston to move the gas phase medium. Because of the excellent wettability of the liquid piston, the contacting inner surfaces of the microfluidic channel get completely wetted, which seals any possible tiny leaky spots. From our newly developed micro pump, by circulating the liquid pistons along the microfluidic channel, a leak-tight air pumping without dead-volume has been clearly observed.
\end{abstract}

\section{INTRODUCTION}

A low backflow in a pump indicates high pumping efficiency, which is desirable especially for a micro gas pump. Though many scientists have been working on various types of micro gas pumps[1], a micro gas pump without backflow has hardly been achieved. The differently unique properties of gas as compared to liquids have made obtaining high pumping efficiencies extremely hard. Especially, it is very difficult to overcome the high compressibility of gases[2].

There are various types of micro gas pumps utilizing differently generated forces. A pressure driven mechanical force exerted in the plunger (solid, liquid or gas) can be used to interact with the target gas. Based on the phase of the plunger material for gas pumping, micro gas pumping with the solid plunger has been normally achieved through large diaphragm-based plunging interfaces actuated piezoelectrically[3], electrostatically[4], thermally[5], pneumatically[6, 7] and electromagnetically[8]. In addition, a gas pump with the gas-phase plunger utilizing vapor jet [9] has been introduced. Unlike many conventional methods, we adopted a different approach to achieve an effective gas pumping method with a simple structure. So far, the use of the liquid plunger for gas pumping has not been fully explored yet. Although the formerly used ferromagnetic fluid (liquid plunger) with large multiple external magnets has been used for liquid pumping application, it is not appropriate for gas pumping[10]. For our new gas pump, many interesting properties of low surface energy fluids have been considered because high surface energy fluids cannot wet the corner of the microfluidic channel but always leave some void spots causing gas leakage. The low surface energy fluid can effectively wet all the corners of the microfluidic channel very easily and completely[11]. Thus, it can be an effective plunger material for the gas pumping in the microfluidic channel. Moreover, the size of our pump has a great potential to be truly miniaturized since it requires no external large magnets but small electrodes.

\section{DESIGN AND THEORY}

Oil, which is one of the most common low surface energy liquids, was looked into and silicone oil was chosen as the liquid piston material. To drive the silicone oil droplet (a good dielectric material) in a microfluidic channel, we utilized the dielectrophoresis (DEP) force which can be directly imposed to the droplet. The dielectrophoretic body force is generated by the electric field and dominantly contributes to the motion of the dielectric liquid piston in the microfluidic channel. The motion of the liquid can also be further explained by the Navier-Stokes equation. In the equation, the body force density induced by the external electric field term can be expressed by the KortewegHelmholtz electric force density $[12,13]$ :

$$
\mathbf{f}_{\mathbf{b}}=\rho_{\mathrm{f}} \mathbf{E}-\frac{1}{2} \mathrm{E}^{2} \nabla \varepsilon+\nabla\left(\frac{1}{2} \mathbf{E} \cdot \mathbf{E} \frac{\partial \varepsilon}{\partial \rho}\right)
$$

where $\varepsilon$ is the fluid permittivity, $\rho$ is the density of the fluid, $\rho_{\mathrm{f}}$ is the free electric charge density, and $\mathrm{E}$ is the electric field. By ignoring the electrostriction and assuming the ideal dielectric conditions for the liquid piston, the body force can be further simplified as a proportional expression:

$$
\mathbf{f}_{\mathbf{b}} \propto E^{2} \nabla \varepsilon
$$

From this equation, we can notice that the DEP force increases especially at the region where the permittivity gradient is maximized. In addition, with an increase of the electric field applied across the gradient areas, the body force density will also be increased. Since we have used a fixed channel height in our design, the electrical field is strongly correlated to the actuation voltages.

\section{FABRICATION AND EXPERIMENT Single Liquid Piston}

To begin with, a single liquid piston in a straight microfluidic channel was tested for characterizing the pumping capability. As illustrated in Fig. 1, the straight channel device consists of two main parts: 1) A PDMS microfluidic channel, and 2) Parallel-plate electrodes for providing the electric field along the microfluidic channel. Fabrication of the device was very simple. First, a thin aluminum film was sputter-deposited and patterned to form electrodes. Along the electrode pads, a microfluidic channel was built by using patternable PDMS. A thin indium-tin-oxide (ITO) coated glass substrate was used as the top cover because it can provide clear visibility for careful observation of the liquid piston behavior from various tests. Finally, the bonding was achieved between top and bottom structures by oxygen plasma treatment on the PDMS surface.


Figure 1. Schematic drawing of a single liquid piston kept in a straight microchannel with a small contact angle liquid (left) and microscope image of the fabricated device (right). 
A small silicone oil droplet was loaded from an open end of the channel with the aid of an external pneumatic source. Due to the very low surface tension of silicone oil, the contact angle on the PDMS microfluidic channel surface turned out to be far less than $90^{\circ}$. From the top view of the device as shown in Fig. 1, we can clearly see the concave edge profiles from both the sides of the silicone oil droplet, which resulted from good wettability over the inner surfaces of the microfluidic channel.

Then, the device was placed on a test platform and connected to a computer-controlled National Instruments (NI) digital I/O device, which allows us to handle a variety of driving signal patterns. The results show that the liquid piston motion closely follows the actuation signals. When the driving signal is applied to an electrode, a gradient of permittivity on the front edge line of the liquid piston is generated. It was observed that the liquid piston responded rapidly to the applied signal. When the electrode is activated sufficiently long enough for the liquid to sweep across the entire actuated area, the front edge of the moving liquid piston stops at the front edge of the leading electrode. The movement of the liquid piston is quick and highly electrically controllable. The characterization is based on the sweeping frequency and the average speed of the liquid piston. The backpressure of a single liquid piston can be improved by increasing the actuation voltage as shown in Fig. 2. The corresponding flow rate to the driving signal sweeping frequency is characterized.


Figure 2. Characterization data of a single liquid piton. A) Backpressure vs. voltage. B) Speed and flow rate vs. driving signal sweeping frequency.

\section{Micro Gas Pump}

Based on the results of a single liquid piston experiment in a microfluidic channel, we have designed and developed a micro gas pump generating continuous flow. Different from the single liquid piston device, the micro gas pump consists of three important functional parts: 1) A closed microfluidic channel for guiding liquid pistons, 2) An array of electrodes placed along the fluidic path in the microfluidic channel for generating the actuation force on the liquid pistons, and 3) An isolation stage between inlet and outlet ports. The isolation stage in the micro gas pump, especially plays a very important role. It holds a sufficient amount of the liquid for multiple liquid pistons. In addition, within the isolation stage, high fluidic resistance is needed to avoid unwanted backflow through the short path between the inlet and outlet ports of the pump. For this, we have employed semi porous structures for increasing the fluidic resistance in the isolation stage. Low surface energy liquids can easily wet the semi porous structures providing the significantly increased surface-to-volume ratio and capillary force.

First, we fabricated, tested and characterized the isolation stage with semi porous structures to investigate its effectiveness. For this, a spin coated SU8 layer was etched by reactive ion etching (RIE) to make the structure semi porous. Fig. 3 shows lots of small semi porous SU8 features generated by oxygen RIE. After being assembled within a microfluidic channel, a silicone oil droplet was loaded in the semi porous structure.


Figure 3. A) Optical and B) SEM images of SU8 semi porous structure for the isolation stage.



Figure 4. Snapshots of the backpressure testing for straight channel with modified semi porous SU8 (marked in red square box) structure working as isolation stage. Dash lines represent the inner surfaces of microchannel. Inlet pressure P1 kept increasing during the testing, while P2 kept constant.

An external pressure source was connected to the microchannel and an external pressure sensor through a $\mathrm{T}$ connection. The motion of liquid piston was captured through a CCD camera while monitoring the pressure recorded through a LabVIEW controlled pressure sensor. An increasing pressure was built up inside the microchannel and the liquid piston was monitored till it burst open. The threshold pressure was measured 
from this test. The liquid was seen to remain stationary until it reaches the threshold breaking pressure. Since the semi porous SU8 structures had a thickness of around $10 \mu \mathrm{m}$, addition of the structure reduces the channel height in the isolation stage. It has been observed that the semi porous SU8 structure held the silicone oil strongly and significantly increased the overall backpressure of the device up to $6 \mathrm{kPa}$. If the porosity of the structure is increased, we believe that the backpressure will also be improved.
A

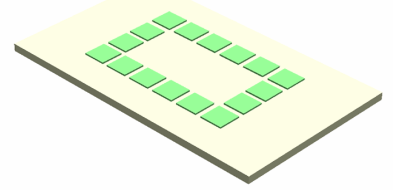

C





D



Figure 5. Brief fabrication steps, A) An array of electrode pads (green) fabricated on top of glass substrate. B) PDMS microchannel (purple) built on top of it. C) Semi porous SU8 structure (orange) was fabricated on top of cover ITO glass. D) The overview of entire device after oxygen plasma bonding.

Fabrication of the micro gas pump is very similar to that of the single liquid piston device as illustrated in Fig. 5. Like previously, an ITO coated cover glass was also utilized as a top substrate (ground) for careful monitoring of the pump. The semi porous SU8 structure was added between the inlet and outlet ports for the isolation stage. Once the fabrication was completed, the micro gas pump was mounted on the testing bench and controlled by the Labview codes. As the signal circulates, one or more liquid piston(s) can move forward and push air through the microfluidic channel. Between the liquid pistons, an air bubble can be caught and carried towards the output of the pump. Since the semi porous SU8 structure increases the liquid sorption property, it can stably hold the liquid piston at the isolation stage. Due to the delectrophoresis force acting downwards and contour force from isolation stage force acting upwards the liquid droplet can maturely split. To facilitate trapping of the gas bubble near the inlet, the duration of the applied actuation signals was intentionally increased. Moreover, the electrode pads near the inlet were designed in a triangular shape (electrode \#3, 4 in Fig. 6) to ease the transition of the gas bubble from the inlet into the circular pumping zone. During the following cycle, when the electrodes (electrode \#2-4) near the inlet port are activated to the 'ON' state again, another liquid piston is pulled from the isolation stage and fills in the microfluidic channel. The fill-in force is sufficient to bridge the gap at the inlet port. In this way, the air bubble gets trapped. In the rest of the cycling process, the liquid piston is driven electrically and moves clock-wise for carrying the air bubble towards the outlet. The liquid piston merges back into the isolation stage at the outlet, and shifts towards the inlet again.

The silicone oil imbibed semi porous structure plays the dual roles of routing the liquid back towards the inlet while maintaining sufficient backpressure. It selectively absorbs silicone oil as compared to gas; the oil gets wicked in while the gas bubble is repelled. In other words, the isolation stage acts as a gas-repelling structure, preventing the gas from back-flowing into the loading zone near the inlet.


Figure 6. A) The electrode pads (pink) numbered in a clockwise sequence from the center top one. B) Actuation signals on each of the electrode pads on right.

Moreover, liquid piston movement along the circular pumping zone microchannel was tested continuously. Firstly, the speed limit of a single droplet was characterized along the clockwise path of the circular pumping zone without pressure load (zero backpressure). The average speed followed by the single droplet testing resulted in a sweeping frequency of $2.5 \mathrm{~Hz}$. We have found that the average speed of the droplet was heavily dependent on the clearing time of the outlet port and can be improved if the liquid piston can be absorbed by the isolation stage and clear the outlet port quickly enough. In other words, the silicone oil needs to be routed back to the inlet as fast as possible. A slow motion approach can be used to guarantee a successful pumping stroke in our prototype stage. A series of actuation signals were programmed using LabVIEW code. By controlling the driving signal applied to each electrode pad (Fig. 6.), a successful cyclic pumping was achieved as each step is clearly shown in Fig 7.



Figure 7. The continuous pumping results shown in sequenced snapshots. The pumping direction is marked in red arrows and trapped gas bubble is marked in dashed red circle.

The flow rate for our device is related to the circulating speed of the pistons, and the volume of the gas bubble captured in each 
cycle. During the cyclic pumping process, an adjustment of the time interval between the two liquid pistons at the inlet port directly affects the size of the trapped gas bubble in between. Therefore, by adjusting signals near the inlet port, the average volume of the trapped gas bubble can be determined. Post image analysis was adapted to get the volume information of the trapped bubble. The air bubble is always generated from the upper boundary of electrode \#4 as shown in Fig. 7B F and the volume of the trapped air bubble is calculated from the electrode area under the air bubble.

Based on the total volume of the trapped gas bubble per cycling time, flow rate can be further calculated. As shown in Figure 8 , the controllability of the trapped air bubble size turns out to be very good. The sizes of the trapped air bubble at the inlet are very close to what we planned to capture.



Distance from inlet (number of electrode pad)

Figure 8. The size of both planned and captured air bubble during the continuous pumping.

\section{CONCLUSION}

We have successfully demonstrated a novel micro gas pumping technique with liquid pistons. By utilizing a low surface tension liquid as working fluid (silicone oil), a complete wetting of the inner surfaces of the microfluidic channel successfully provided leak-tightness and effectively eliminated gas leakage problems including high dead-volume and backflow. The electrokinetically driven liquid piston made the pumping process highly controllable. To achieve a higher backpressure and separation between gas and liquid media, we utilized semi porous SU8 structures as an isolation stage, which has been proven to provide sufficient gas-repelling force and to increase the backpressure. This micro gas pump may bring a promising way to the efficient micro gas pumping mechanisms.

\section{REFERENCES}

[1] P. Woias, "Micropumps--past, progress and future prospects," Sensors and Actuators B: Chemical, vol. 105, pp. 28-38, 2005.

[2] M. Richter, R. Linnemann, and P. Woias, "Robust design of gas and liquid micropumps," Sensors and Actuators A: Physical, vol. 68, pp. 480-486, 1998.

[3] H. Van Lintel, F. Van de Pol, and S. Bouwstra, "A piezoelectric micropump based on micromachining of silicon," Sensors and Actuators, vol. 15, pp. 153-167, 1988.

[4] T. Bourouina, "Design and simulation of an electrostatic micropump for drug-delivery applications," Journal of micromechanics and microengineering, vol. 7, p. 186, 1997.

[5] F. Van de Pol, H. Van Lintel, M. Elwenspoek, and J. Fluitman, "A thermopneumatic micropump based on micro-engineering techniques," Sensors and Actuators A: Physical, vol. 21, pp. 198-202, 1990.

[6] C. W. Huang, S. B. Huang, and G. B. Lee, "Pneumatic micropumps with serially connected actuation chambers," Journal of micromechanics and microengineering, vol. 16, p. 2265, 2006.

[7] H. Zhou, H. Li, V. Sharma, and M. Schmidt, "A single-stage micromachined vacuum pump achieving 164 torr absolute pressure," 2011, pp. 1095-1098.

[8] C. Yamahata, C. Lotto, E. Al-Assaf, and M. Gijs, "A PMMA valveless micropump using electromagnetic actuation," Microfluidics and Nanofluidics, vol. 1, pp. 197-207, 2005.

[9] M. Doms and J. Mueller, "A micromachined vapor jet pump," Sensors and Actuators A: Physical, vol. 119, pp. 462467, 2005.

[10] A. Hatch, A. E. Kamholz, G. Holman, P. Yager, and K. F. Bohringer, "A ferrofluidic magnetic micropump," Microelectromechanical Systems, Journal of, vol. 10, pp. 215-221, 2001.

[11] J. Jiao, "Gas Pumping Effect from Dielectrophoretically Driven Liquid Droplet in a Closed Microfluidic Channel," M.S. Thesis, University of MissouriColumbia, USA, 2010.

[12] T. Jones, "Liquid dielectrophoresis on the microscale," Journal of Electrostatics, vol. 51, pp. 290-299, 2001.

[13] P. M. Young and K. Mohseni, "Calculation of DEP and EWOD forces for application in digital microfluidics," Journal of Fluids Engineering, vol. 130, p. 081603, 2008.

\section{CONTACT}

*J.Jiao, tel: +1-573-884-7867; jjw94@mail.missouri.edu 